\title{
Initiation propagation and termination of elastodynamic ruptures associated with segmentation of faults and shaking hazard
}

\author{
Bruce E. Shaw ${ }^{1}$ \\ Received 7 October 2005; revised 13 March 2006; accepted 12 April 2006; published 10 August 2006.
}

[1] Using a model of a complex fault system, we examine the initiation, propagation, and termination of ruptures and their relationship to fault geometry and shaking hazard. We find concentrations of epicenters near fault step overs and ends; concentrations of terminations near fault ends; and persistent propagation directivity effects. Taking advantage of long sequences of dynamic events, we directly measure shaking hazards, such as peak ground acceleration exceedance probabilities, without need for additional assumptions. This provides a new tool for exploring shaking hazard from a physics-based perspective, its dependence on various physical parameters, and its correlation with other geological and seismological observables. Using this capability, we find some significant aspects of the shaking hazard can be anticipated by measures of the epicenters. In particular, asymmetries in the relative peak ground motion hazard along the faults appear well correlated with asymmetries in epicentral locations.

Citation: Shaw, B. E. (2006), Initiation propagation and termination of elastodynamic ruptures associated with segmentation of faults and shaking hazard, J. Geophys. Res., 111, B08302, doi:10.1029/2005JB004093.

\section{Introduction}

[2] The faults on which earthquake occur are not simple planar structures, but have bends, jogs, branches, and steps in them. These geometrical irregularities have been proposed to play important roles in a number of aspects of earthquake behavior, including rupture initiation and termination [King and Nabelek, 1985]. While many observed ruptures support the role played by geometrical irregularities in rupture initiation and termination [King and Nabelek, 1985], other examples pose questions for simple interpretations of the roles of segmentation in delineating earthquake ruptures: The 1992 M7.1 Landers event which initiated in the middle of one segment, jumping two segment step overs and then dying in the middle of a third segment is one such example. Clearly, a more thorough understanding of the role of geometrical irregularities in earthquake dynamics is needed.

[3] The observational data remains fundamentally limited by the long repeat times between large events; the short timescales of the instrumental record relative to the repeat timescale of large events means we typically have at most one rupture recorded on a given fault (the exception in the case of the Parkfield segment, where four events have been recorded, shows how generally true this is). Finding systematic patterns on a given fault geometry, and seeing how to average across different fault geometries then becomes difficult. In this context, numerical simulations of geomet-

\footnotetext{
${ }^{1}$ Lamont-Doherty Earth Observatory, Columbia University, Palisades, New York, USA.

Copyright 2006 by the American Geophysical Union. 0148-0227/06/2005JB004093\$09.00
}

rically complex fault systems can play a tremendously useful role; they provide long catalogues so patterns as well as variability can be seen, as well as privileged access to stress fields and other relevant information that can help elucidate physical bases for the patterns.

[4] Previous work has examined the evolution of populations of events on complex fault systems; these approaches have, however, neglected the dynamics on the rupture timescale, by simplifying the interactions to be quasi-static [Lyakhovsky et al., 2001]. Other models have treated elastodynamic event populations, but only with simple fault geometries [Carlson and Langer, 1989; Myers et al., 1996; Cochard and Madariaga, 1996]. A number of models have examined individual elastodynamic events on nonplanar fault geometries, but not populations of events [Harris et al., 1991; Kame and Yamashita, 1997; Bouchon and Streiff, 1997; Aochi et al., 2000]. Two modeling efforts have looked at event sequences on an individual complex fault [Mora and Place, 1999; Duan and Oglesby, 2005]. Our work is new in providing long catalogues of elastodynamic ruptures on geometrically complex fault systems [Shaw, 2004a, 2004b]. With these new kinds of catalogues we examine here questions of the role of segmented fault geometry in rupture initiation, propagation, and termination, and the impact on shaking hazard.

[s] We present a new tool for exploring earthquake hazard in a physics-based model. While these models do not have the complete set of earthquake behaviors, they are nevertheless rich enough to use in a variety of ways. We can, for example, compare and hone our techniques and pose new questions in a context where limited catalogues and statistics are not an issue [Pepke et al., 1994] before tackling real catalogues [Kossobokov and Carlson, 1995]. 
Here, we examine two new capabilities of dynamic models, the locations of epicenters, and the peak ground shaking; taking this even further, we find a significant correlation between them.

\section{The Model}

[6] The model is meant to capture the behavior of fault systems in map view, being a simplified two-dimensional projection of the brittle crust, averaged over the depth direction. It thus misses aspects of three-dimensional fault behavior, such as the asymmetry between the hanging wall and footwall [Brune, 2001; Oglesby et al., 1998]. It does, however, capture a richness of along-strike variations resulting from stress enhancement and shadows in the interaction of faults. Furthermore, the two-dimensional simplification allows us to simulate very long catalogues over the whole fault system, with tens of large event cycles occurring on each fault.

[7] The model consists of a scalar two dimensional brittle upper layer coupled to a slowly loaded ductile lower layer. When the stresses in the brittle layer exceed the strength, dislocations occur. All of the nonlinearity in the problem comes from how the strength evolves. It begins from some initial unbroken strength having some overall value plus a spatially uncorrelated random component. A long term geological slip weakening localizes the slip onto faults and leads to a slow geological evolution of the fault system [Spyropoulos et al., 2002]. Dynamic weakening during slip events leads to sudden stick-slip events. A variety of dynamic weakening mechanisms are explored, including slip weakening, velocity weakening, and time weakening [Shaw, 1997; Shaw and Rice, 2000]. We explore a range of frictions because the frictional behavior at seismic slip rates remains a fundamental open question. The model, and the equations defining it have been presented elsewhere [Shaw, 2004a, 2004b], although only in complete form in an electronic supplement; we thus present the full model equations and a discussion of the numerics here in Appendix A. We use slip weakening for the dynamic weakening as the default friction in the figures, but other frictions, including velocity weakening, give similar results. Lengths in the problem are scaled to the seismogenic depth, which has been scaled to unity. Appendix A presents a listing of conversions from dimensionless units, which we use throughout the paper, to dimensional units, to aid in comparing the results with earthquake observations.

\section{Results}

[8] Beginning from some stage in the slow geological evolution of the fault system, we examine a long sequence of elastodynamic ruptures. These ruptures display a rich complexity of behaviors.

[9] Figure 1 shows three different views of the complex sequences which develop, and the long catalogue of dynamic ruptures which occur on it. Figure 1a shows the slip rate on each of the faults, grey scale proportional to slip rate. We see very interesting patterns of linked segments, and smaller faults associated with large segment step overs and ends. These smaller faults help accommodate deformation, keeping stresses finite with accumulating strain.
[10] Before continuing, we should discuss how realistic the model fault system geometries are. It is important to understand that we are not imposing a geometry on the fault system but rather are letting a physics that we propose, a geological slip weakening, localize the deformation onto its own fault system. Thus the system self-consistently accommodates strain as it chooses, and no singularities develop over time (if stress concentrations grow, they simply break a new fault). We are also, however, imposing some substantial numerical constraints. The faults grow on a lattice, breaking a minimum fault length $\delta_{x}$, with a minimum separation in the perpendicular direction from other faults of distance $\delta_{y}$. For numerical simplicity, we further constrain the faults to break only along one direction, which then limits the geometric irregularities considered to just fault segmentation based on step overs, with bends and jogs thus not accounted for. Nevertheless, the complex step over geometry displays many interesting features which are quite suggestive of real faults. For example, the distribution of segment lengths [Spyropoulos et al., 2002] evolves in ways similar to laboratory analogue systems [Spyropoulos et al., 1999], and normal faults on Venus where the best data set on fault population lengths has been found [Scholz, 1997]. Other aspects of the fault behavior, such as slip distributions along faults and at step overs, look very realistic when compared with field observations [Dawers and Anders, 1995; Manighetti et al., 2005]. Additionally, while our segmentation is limited to arising only from step overs, it has been argued that bends and step overs can play similar roles in impacting fault systems in terms of rupture initiation and termination [King, 1986].

[11] The dynamic ruptures which occur on these complex fault systems have interesting relationships with the fault geometry. Figure 1b shows the density of epicenters for a long sequence of dynamic events on this fault system ( 8 thousand events in the catalogue shown here). Interestingly, the epicenters occur both along the main faults, where slip has been concentrated, and along the minor linking faults off of the main faults where little slip has occurred. Ruptures initiating on these minor faults can sometimes jump onto larger adjacent segments and grow into large ruptures, so they play a nonnegligible role in the hazard. Distributions of sizes of events on the fault systems, and their relationship to segment lengths, have been examined previously [Shaw, 2004a].

[12] Figure $1 \mathrm{c}$ shows the density of rupture terminations, where the two terminations of each rupture are defined as the farthest points ruptured on either side of the epicenters. They are strongly associated with segment ends, and are even more concentrated than the epicenters. (The concentration of the density $\rho, \sqrt{\left\langle\rho^{2}\right\rangle /\langle\rho\rangle^{2}}=96$ for terminations, 51 for epicenters, 20 for slip for the catalogue in Figure 1). [13] The epicenters also have a strong association with the fault geometry. We see them happening toward the ends of the segments, near segment step overs, as well as over a broader area of smaller faults. In contrast, interiors of large segments tend to be relatively less populated with epicenters. Similar effects are seen in examples on real faults, for example along the San Andreas fault which ruptured in the great 1857 earthquake, which is today seismically very 

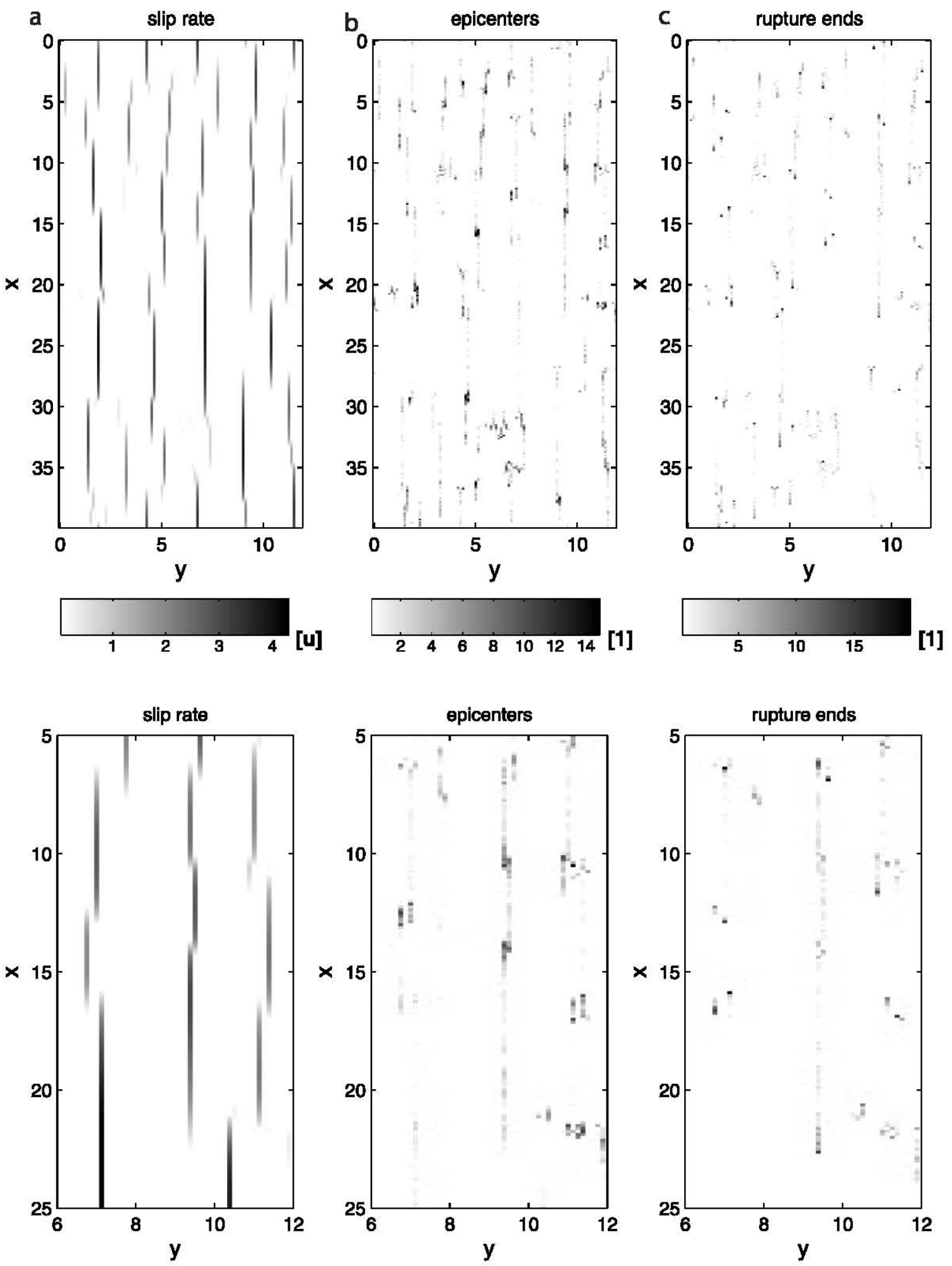

Figure 1. Three views of a long catalogue of events. (a) Slip rate per unit strain. (b) Epicentral density. (c) Rupture termination density. Note the association of epicenters with segment step overs and ends and the association of rupture terminations with segment ends. Numbers on the horizontal and vertical axes are distances in units of the brittle crust depth, corresponding to unscaled lengths of order a few hundred kilometers across by a thousand kilometers long. Scale bars indicate grey scale levels. Brackets on scale bars indicate units. (bottom) Blowup detail of Figure 1 (top). 
(a)

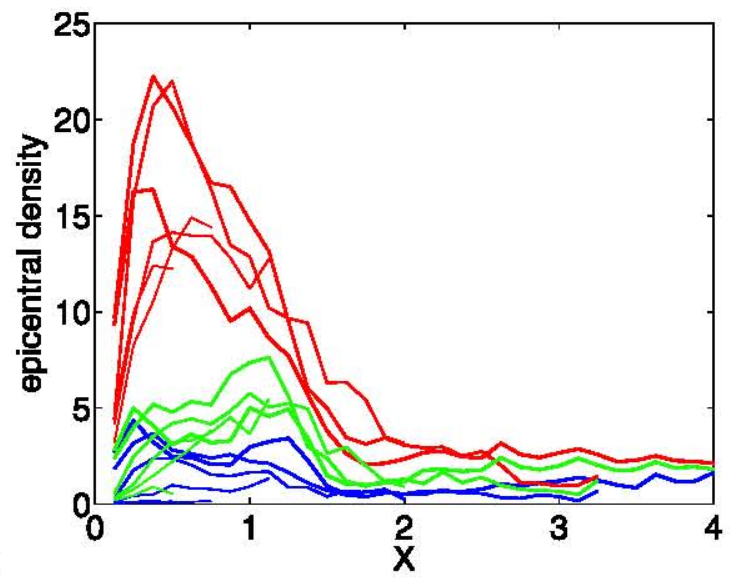

(b)

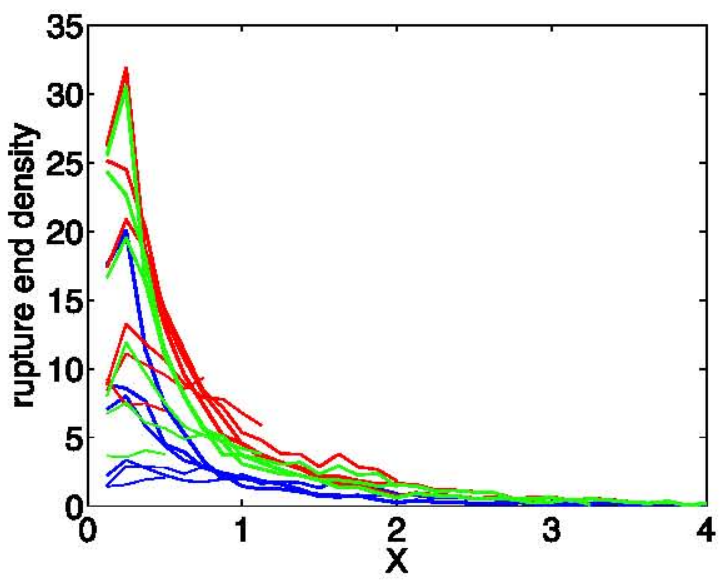

Figure 2. Stack of locations of event epicenters and ends occurring at distance $X$ from segment ends. (a) Epicentral density. (b) End density. Thicker lines correspond to longer segments. Note the rate per segment is very insensitive to the segment length, indicated by the overlapping of the lines of different thickness. The top red lines are for rupture lengths including and above the smallest events; the middle green lines are for events which ruptured length unity and above, corresponding to large events, while the lower blue lines are for events rupturing above length 8 , corresponding to very large events. Note that the epicenters of large events tend to nucleate farther from the segment ends relative to the small events, seen by the fractional area under the green and blue curves being farther from the segment ends relative to the red curves.

quiet, in contrast with the San Jacinto fault with is both geometrically more dense in step overs [Stirling et al., 1996] and seismicity. To make the statement about the location of the epicenters and terminations more precise, we can stack their locations relative to the ends of the segments. Figure 2 shows the distributions as a function of distance from the segment ends, grouped by segments of different lengths denoted by lines of different thickness. We look as well at events with different lower cutoff rupture sizes, to examine any size dependence of where the events are initiating. Here sizes is measured by the total length of faults ruptured in an event. Distance is measured by how far away from the segment end the event initiated, and how far away from the segment end the event terminated, denoted by the distance $X$. In Figure 2, we keep track of how long the rupture event was by plotting events of different rupture lengths with different colors. We see little segments length dependence to where events are initiating, evidenced by the similar behavior of the different thickness lines of the same color. Interestingly, we see some magnitude dependence of where events are initiating: Large events tend to have epicenters farther from the segment ends as compared with the small events, evidenced by the green and blue curves having a bigger fraction of their area farther from the segment ends, bigger values of $X$, than the red small event curves. This means we have a significant difference in the magnitude distribution depending on the location of initiation of events. Figure $2 \mathrm{~b}$ showing the stack of ends of events shows little segment length or magnitude dependence; most, but by no means all, events end at segment boundaries, with probabilities falling off exponentially with distance from the ends.

[14] In addition to the initiation and termination, the propagation of ruptures is also strongly influenced by the fault geometry. We see persistent directivity effects on most of the fault system. Figure 3a shows the fraction of ruptures propagating in either direction, color scaled with blue for up and red for down, with the fraction defined by $f=\left(N_{\mathrm{up}}-\right.$ $\left.N_{\text {down }}\right) /\left(N_{u p}+N_{\text {down }}\right)$ so $-1 \leq f \leq 1$. Here $N_{\text {up }}$ is the number of times a point breaks during a rupture event with the rupture locally propagating upward, while $N_{\text {down }}$ is similarly when it is locally propagating downward. Note the excess of extreme directivity values where nearly all the ruptures are propagating in one direction, the peaks at fractions \pm 1 . Compare this to the distribution for the case where initiation sites were uniformly distributed across the segment, and all ruptures broke the whole segment, a simple model that McGuire et al. [2002] present in their discussions of global distributions of unilateral versus bilateral ruptures. That simple model produces a linear increase in density from zero at the center $f=0$ out to a maximum at the ends $f= \pm 1$. Here we have many more places having extreme values of $|f|$ close to unity, with the peaks far above a linear extrapolation from moderate $|f|$ values.

[15] The rupture directivity plots reveal an interesting aspect of the rupture dynamics in events. Ruptures tend to jump segments when the rupture runs into the end of a segment, propagating bilaterally from the initiation point on the adjacent newly rupturing segment [Harris et al., 1991; Fliss et al., 2005]. This shows up clearly in the directivity plots as a change in the sign of the directivity across the segment overlaps. These backward propagating branches of the bilateral ruptures soon die out, however, due to the stress shadows of the adjacent segments.

\subsection{Shaking Hazard}

[16] In addition to giving us insight into rupture dynamics processes, directivity plays an important role in hazard. Rupture directivity makes a big difference in ground motions, amplifying or decreasing motions by several fold, depending on whether the rupture is propagating toward or away from a location [Somerville et al., 1997]. Indeed, we 


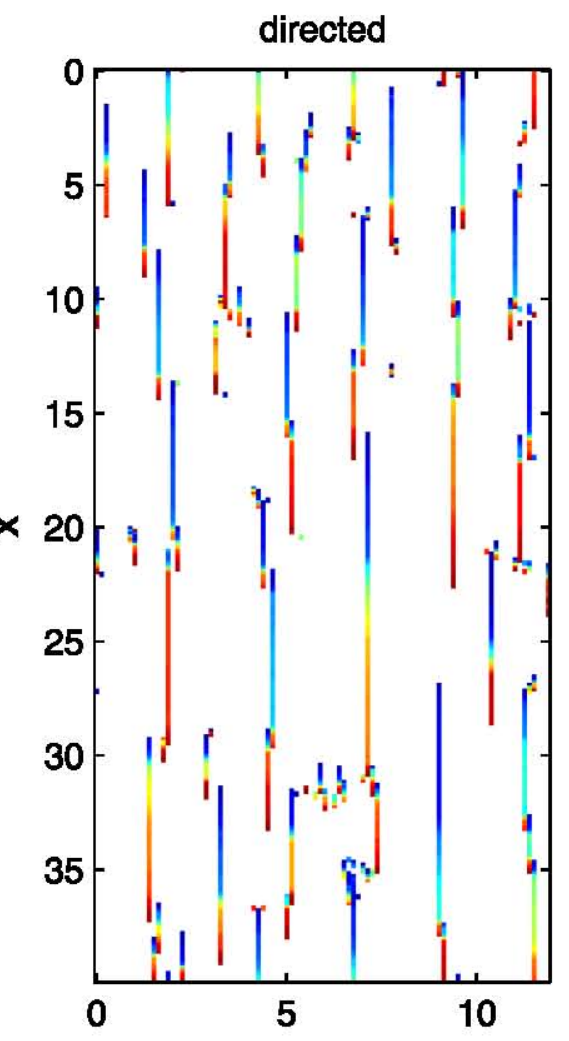

(a)

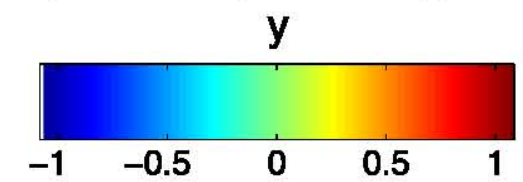

(b)

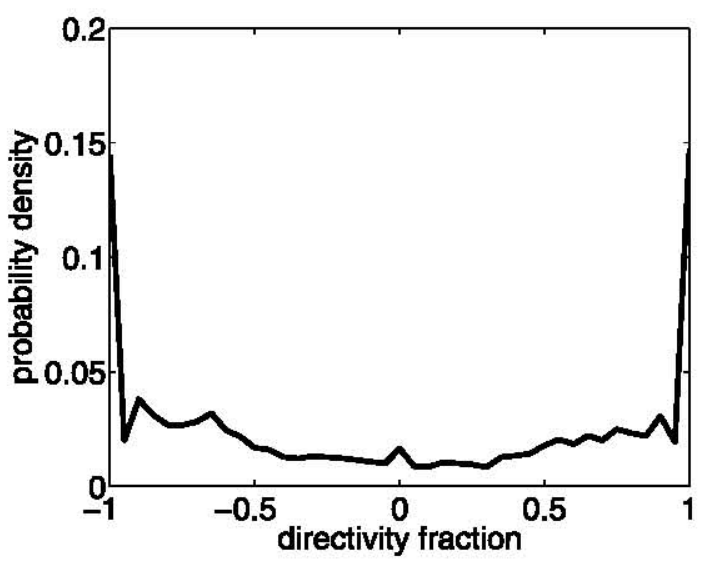

Figure 3. Directivity of ruptures. (a) Fraction of events propagating up (blue colors) versus down (red colors). (b) Histogram of rupture directivities. Note high amplitude; persistent directivity is the typical, rather than exceptional case.

can look directly at some hazard measures of shaking in the model, and see these effects. Taking advantage of the fully dynamic nature of the simulation, we measure directly for each event various shaking measures. Figure 4 shows for an example large event two different measures, the peak acceleration in Figure 4a and the peak velocity in Figure 4b. This event illustrates a number of interesting features. Initiating in the upper right set of faults at a step over, it propagated bilaterally up and down (a behavior which Figure 3a shows is typical of this location on the fault system). We see this in the directivity effects, where the peak acceleration and velocity grow larger along the fault segments away from the epicenter. Interestingly, while the rupture jumped an additional segment in propagating upward toward the top (near location 10 on the vertical axis), the high peak shaking rates were reduced following the jump, and took some time to build up again as the slip propagated along the next segment. Another interesting aspect of the shaking there is the reduced shaking in the small area between where the rupture jumped the step over, a not surprising effect since the sign of the displacement reverses there.

[17] One aspect of this event which is atypical is that it dynamically triggered remotely an additional rupture, which we see in the lower left set of faults. While this remote triggering is not common, it does provide a nice illustration of the dynamic effects present in the model. A lobe of radiation from the lower end of the initial set of rupturing segments points to the triggering region in the middle of the fault segment which was dynamically triggered, beginning rupture as the dynamic stress waves passed by. After triggering, a bilateral rupture grew out, with directivity effects again reflecting this growth away from the center of slip initiation.

[18] This rupture illustrates an interesting aspect of the dynamics in that the peak acceleration and peak velocity are not simply scaled versions of each other. This is most easily seen in Figure 4 (bottom), which show a blowup of Figure 4 (top), illustrating some of the spatial aspects of the shaking in more detail. We see, for example, even stronger segment end effects on the peak acceleration than on the peak velocity. We see, as well, more pronounced interference patterns in the acceleration as compared with the velocity, as relatively higher frequencies contribute to the peak values in the acceleration. Interestingly, however, we will see that when we aggregate across long catalogues of ruptures, the peak acceleration hazard maps and peak velocity hazard maps look remarkably similar. 

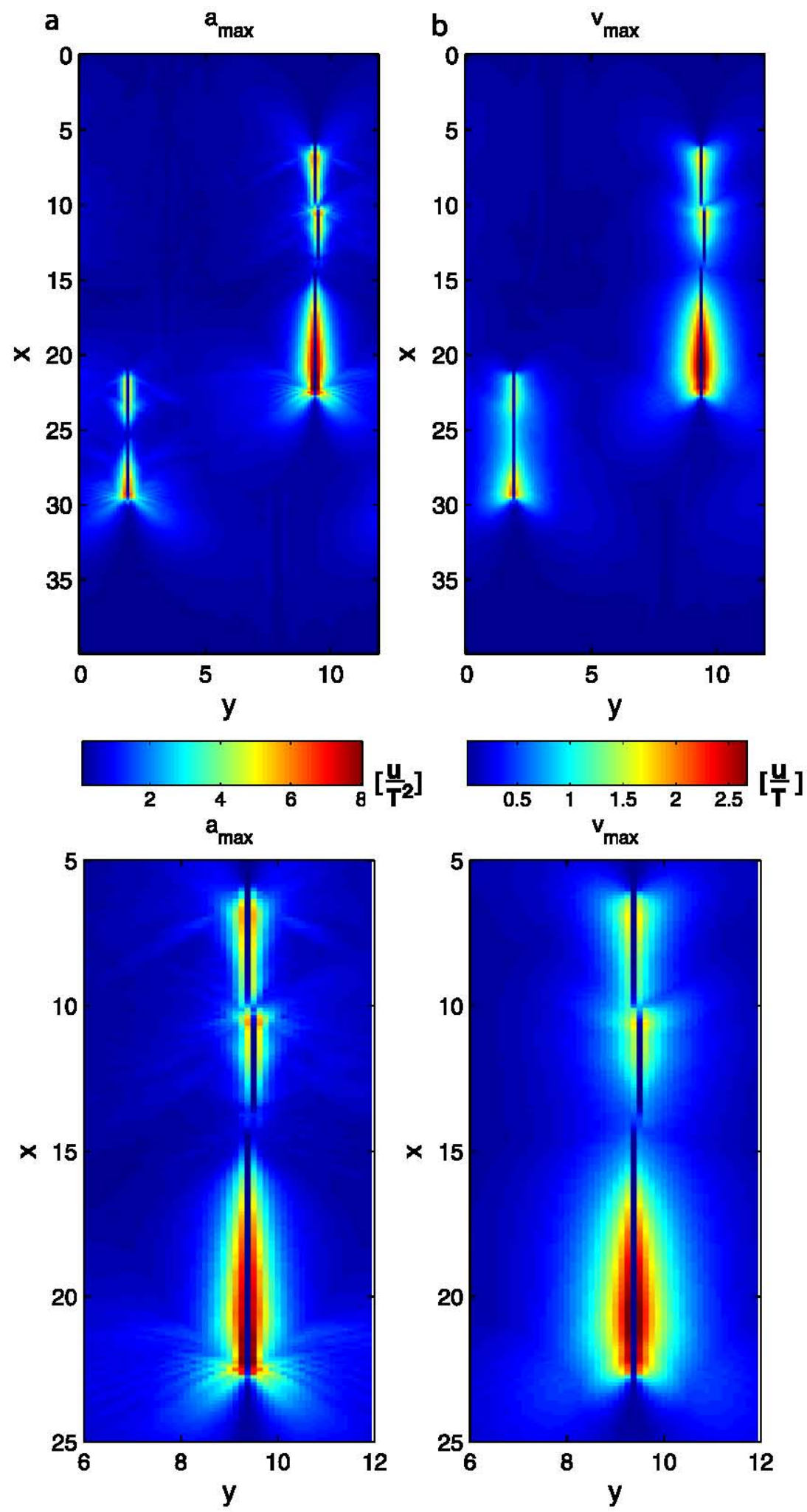

Figure 4. Shaking measures of example large event. (a) Peak acceleration. (b) Peak velocity. Epicenter of event is located at 9.5 horizontal, 14.4 vertical, on left side of step over. (bottom) Blowups of Figure 4 (top) to show more detail of effects of segmentation.

[19] Utilizing a long catalogue of dynamic events, we can make a hazard map for the fault system, combining thousands of dynamic ruptures measured individually as in Figure 4. Figure 5a plots a standard measure of hazard, the peak acceleration for a given probability of occurrence. We measure this in the model by keeping track of peak acceleration at every point in space from each event, combining all the events to make at every point a histogram 

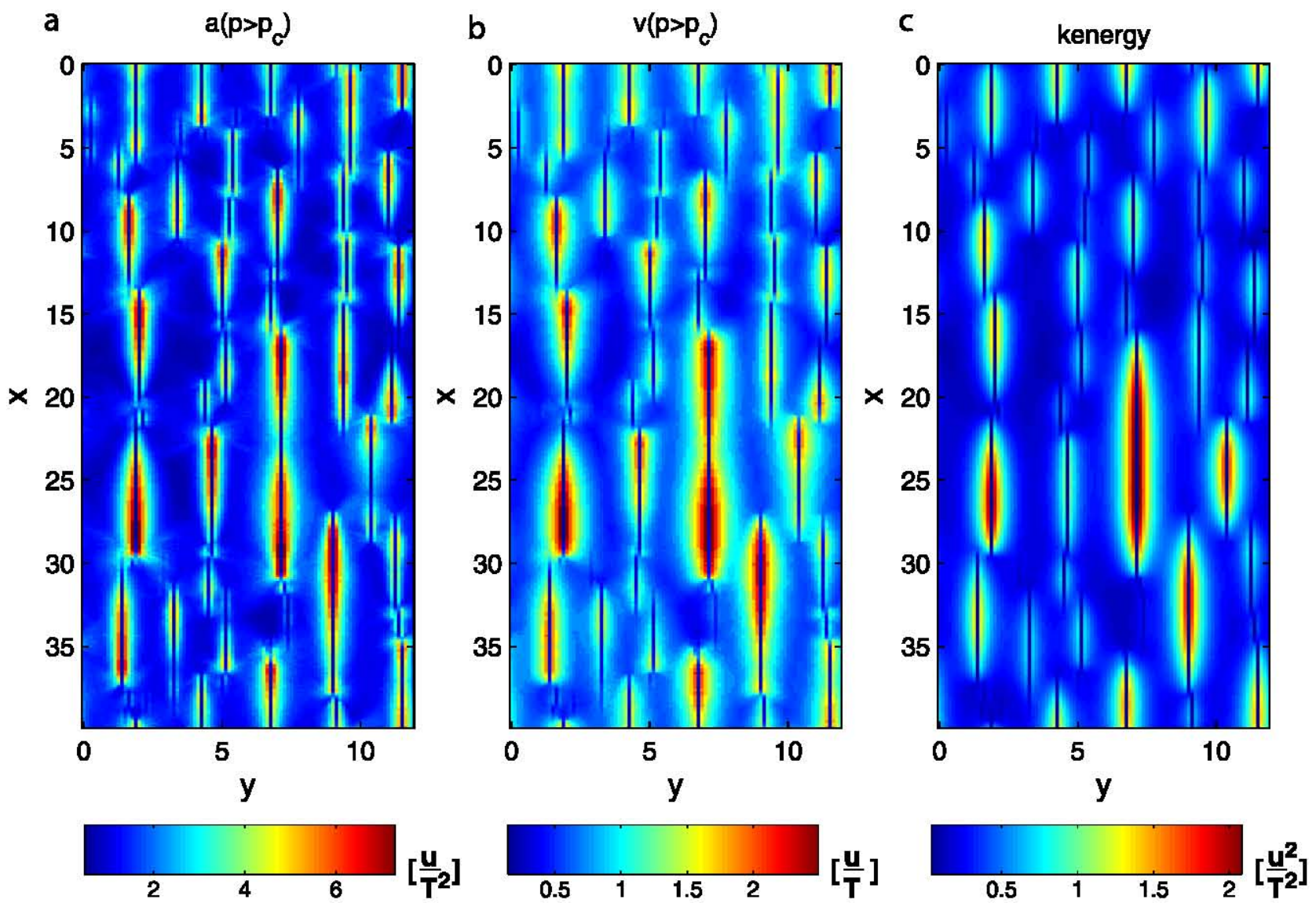

Figure 5. Shaking measures measured directly from long sequence of dynamic events. (a) Peak acceleration for $p>p_{c}$, with $p_{c}=0.2$. (b) Peak velocity for $p>p_{c}$, with $p_{c}=0.2$. (c) Kinetic energy. Note similar maps of peak accelerations and peak velocity, showing significant directivity effects, as contrasted with the more symmetric kinetic energy map.

of these values. Then, choosing a probability value, we plot the acceleration level at which that probability value is exceeded over the long catalogue.

[20] Figure 5b shows the peak velocity for the same probability of occurrence as in Figure 5a the analogue of the $2 \%$ probability of exceedance in 50 year national hazard maps [Frankel et al., 2000] (see also http://eqhazmaps. usgs.gov)). Note the strong similarity with Figure 5a, albeit slightly less concentrated near the faults.

[21] The details of the maps are somewhat thresholddependent; higher cutoff values of probability of occurrence concentrate the probabilities closer to the faults, and weight the larger faults relatively more. Other friction parameter values effects are only slight quantitative, but not qualitative perturbations off of this map. We discuss this point further later in section 3.3 of the paper, and show maps with other parameter values there as well.

[22] The last measure of shaking we show in Figure $5 \mathrm{c}$ is the average of kinetic energy. To make Figure $5 \mathrm{c}$, and Figures $5 \mathrm{a}$ and $5 \mathrm{~b}$, we continue to evolve the dynamics for a significant time beyond when the rupture has come to rest, so the waves have enough time to propagate away from the faults. For Figure 5, after the rupture has stopped, we continue the dynamic simulations for an additional time $T=5$, so the waves propagate an additional distance 5 in every directions away from the fault (doubling the addi- tional simulation time after the rupture stops to $T=10$ reduces the number of events, due to increased computational costs, but does not change the figure significantly). Note, in contrast with the peak motion curves in Figures 5a and $5 b$, the kinetic energy is more symmetric along the fault, and more concentrated along the largest faults. Thus Figure 5c is best anticipated by Figure 1a, the total slip rate on faults. The shaking maps, on the other hand, reflect rupture initiation, propagation, and termination effects in Figures $1 \mathrm{~b}, 1 \mathrm{c}$, and $3 \mathrm{a}$. That the kinetic energy is more symmetric than the peak acceleration or peak velocity is not surprising; it integrates the whole wave train, and thus duration is a factor, which works in the opposite way as directivity, being longer in the backward direction as the rupture propagates away. These two effects do not completely cancel, however, and stronger frictional weakening values leading to larger directivity effects do tend to emphasize the directivity effect more, enhancing the asymmetry in the kinetic energy measurement.

[23] A different way of plotting the results is shown in Figure 6, where we show the probability of a given shaking being exceeded. Interestingly, a plot of peak velocity exceedance probabilities shows a nearly identical map as the peak accelerations map, when the thresholds are chosen so that both have the same total probability of exceedance when integrated over the whole fault system. This scaling 

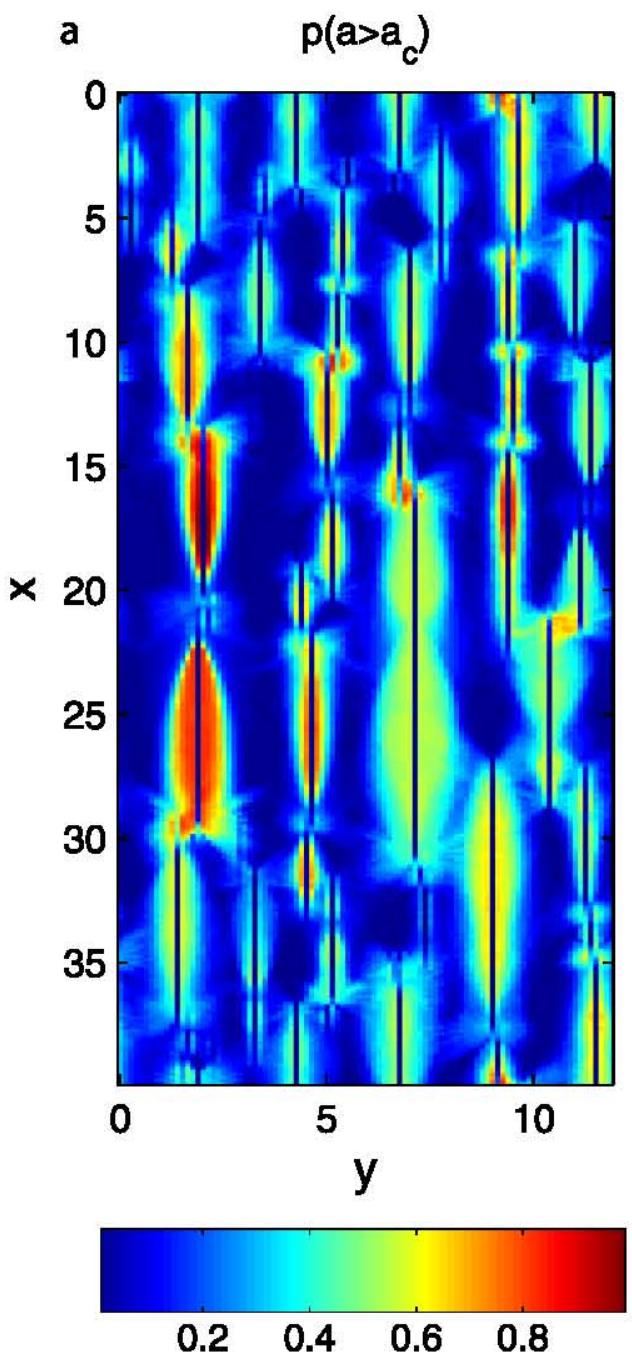

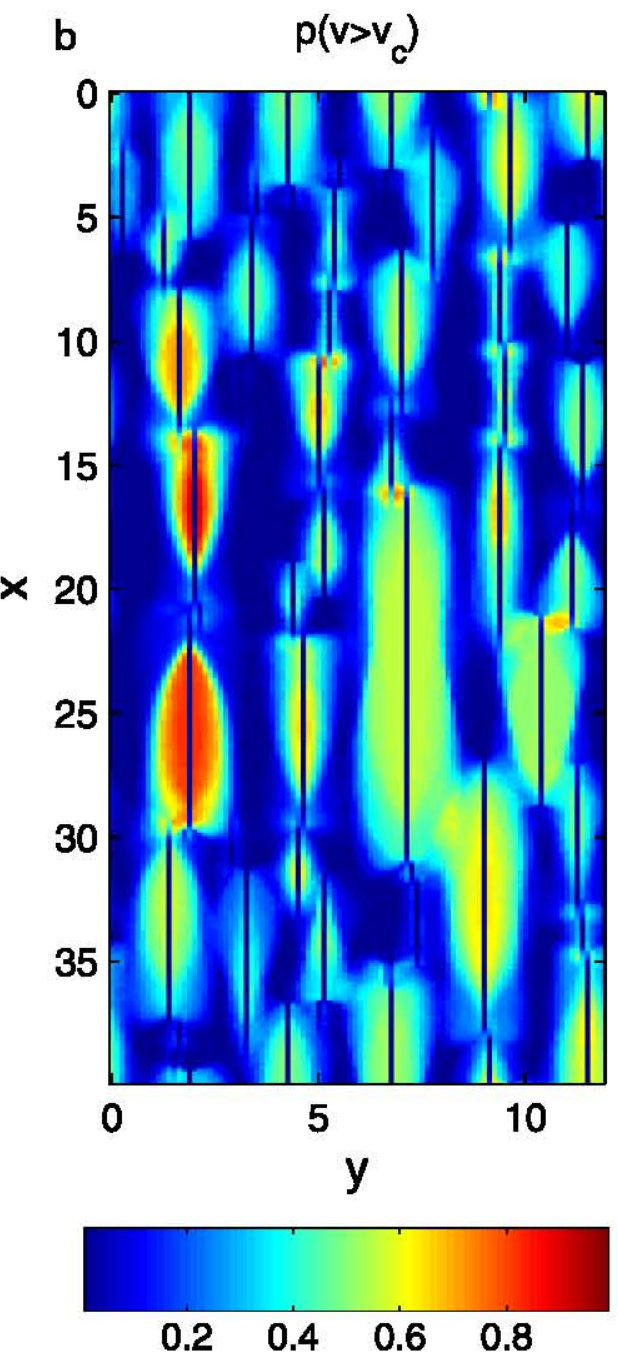

Figure 6. Shaking measures measured directly from long sequence of dynamic events. (a) Probability of peak acceleration exceeding critical acceleration, with critical acceleration chosen to have total average probability of exceedance of $p=0.2$. (b) Probability of peak velocity exceeding critical velocity, with critical velocity chosen to have total average probability of exceedance of $p=0.2$. Note remarkable similarities of the two maps.

occurs despite the differences seen in the spatial pattern for individual events, where peak acceleration and peak velocity can vary substantially with respect to each other spatially, as Figure 4 illustrates. This alternative way of looking at the hazard shows much more interaction between faults, with nearby faults enhancing the hazard since the frequency of large shaking is increased. Segment step overs show up as particularly "hot spots" from this measure. There is also the odd but nevertheless real effect of the largest faults showing a somewhat lowered relative hazard due to the very large but less frequent great events, an effect seen in real faults as well. Since Figure 6 is a less standard way of representing hazard and in addition shows much more variability spatially with changing threshold, we will focus in the rest of the paper on the more standard maps in Figure 5.

\subsection{Correlations of Hazard and Seismicity}

[24] Being able to directly measure the hazard, as opposed to constructing it from parameterizations, we can look for correlations which are not built in. One virtue of having these long synthetic catalogues, on which we can examine details of dynamic ruptures, is we can examine relationships between quantities which may be less easily obtained with observations. Indeed, some significant aspects of the shaking hazard can be anticipated from aspects of the model which have observable geological and seismological analogues. Figure 7 suggests that the asymmetry in the shaking along a fault may be one such measure. We consider two type of asymmetries of a field $f$. The first asymmetry is the average asymmetry $\mathcal{A}(f)$ of the field defined by the difference in the average values of a field along the two halves of a fault segment of length $L$ :

$$
\mathcal{A}(f) \equiv \frac{2}{L}\left(\int_{0}^{L / 2} f\left(x^{\prime}\right) d x^{\prime}-\int_{-L / 2}^{0} f\left(x^{\prime}\right) d x^{\prime}\right) .
$$

The second asymmetry is the distance weighted asymmetry $\mathcal{B}(f)$ of the field defined by the average value of the field 
(a)

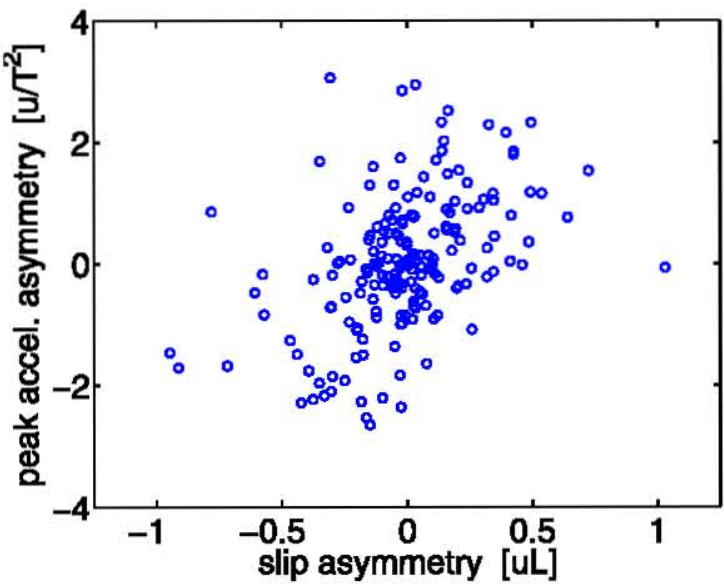

(b)

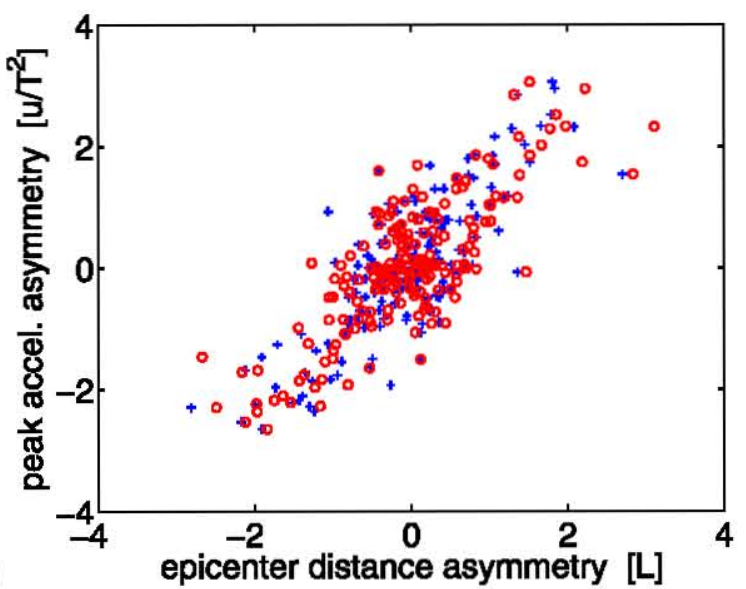

Figure 7. Correlation of asymmetry in peak acceleration on a segment with geological and seismological measures on a segment. (a) Peak acceleration asymmetry versus slip asymmetry. (b) Peak acceleration asymmetry versus epicenter location asymmetry. Blue crosses are using epicenters of small magnitude and larger events, red circles are using epicenters of large events only; both do well at explaining the trend.

weighted by its distance from the center of the segment, which also differences the two halves of the fault, but weights values which are further from the center more. This distance weighted asymmetry is given by

$$
\mathcal{B}(f) \equiv \frac{\int_{-L / 2}^{L / 2} f\left(x^{\prime}\right) x^{\prime} d x^{\prime}}{\int_{-L / 2}^{L / 2} f\left(x^{\prime}\right) d x^{\prime}} .
$$

We use this distance weighting to capture aspects of directivity effects, which increase with propagation distance. Note that while there is some segment length dependence in the distance weighted asymmetry $\mathcal{B}$, since the distance weighting grows with $L$, there is no such effect in the average asymmetry $\mathcal{A}$; thus we will not get spurious segment length correlations if we plot $\mathcal{A}$ asymmetries versus $\mathcal{B}$ asymmetries, as we will do. Note that both of these asymmetries are relative to the segment center: They do not grow just because there is directivity but rather because there is a preferred directivity direction. Thus shaking may be bigger near the ends of the faults just due to directivity, but the shaking asymmetry comes from the difference in shaking on the two halves of the fault.

[25] In Figure 7a, we consider a geological observable, the distance weighted asymmetry in the slip on the two halves of a fault segment. Such asymmetries could be most easily observed in well exposed fault systems [Manighetti et al., 2001], but are not beyond the capability of advanced modeling efforts in places such as southern California. We plot for each segment the slip asymmetry on the horizontal axis and the peak acceleration average asymmetry on the vertical axis (because, by symmetry, the fault itself is a nodal plane for the radiation, the acceleration is measured right next to the fault on both sides and averaged over the two sides). We find some correlation, with $R^{2}=0.45$, so that nearly half of the variance of this shaking hazard asymmetry is accounted for by a geological measure. (While it can be difficult to get the multiple slip measurements this measure requires in some faulting geometries, such as strike-slip environments, other geometries such as normal fault systems provide evidence in the topographic relief.) This asymmetry measure works for the same reason articulated by Manighetti et al. [2005], that events tend to initiate more at the segment step overs, where the fault slip gradients are high, relative to the free unpinned less tapered ends [Ward, 1997].

[26] We can do even better with a seismological observable, the asymmetry in the position of epicenters. In Figure $7 \mathrm{~b}$ we plot on the horizontal axis the average position of the epicenters relative to the center of the segment, and on the vertical axis the same acceleration asymmetry as before [that is, the acceleration average asymmetry $\mathcal{A}\left(a_{\text {peak }}\right)$ versus the distance weighted asymmetry of the epicentral density $\left.\mathcal{B}\left(\rho_{\mathrm{epi}}\right)\right]$. Again we see a strong correlation, this time with an even higher $R^{2}=0.79$. This correlation occurs because of directivity effects: The highest accelerations occur as a large rupture is moving toward you, and since ruptures propagate away from their epicenters, the shaking near the epicenters is lower than the shaking farther down the fault. (Measurements from the dense array of recordings from the recent 2004 Parkfield M6.0 earthquake illustrate well this dumbbell shaking pattern with lower epicentral shaking [Graizer and Shakal, 2004].) We have checked this correlation with a range of lower magnitude cutoff values for the seismicity, shown with different symbols in Figure $7 \mathrm{~b}$ and found similar results independent of the magnitude cutoff. This is good news, suggesting we may be able to use the spatial distribution of the numerous small earthquakes, together with mapped fault geometries, to anticipate statistical aspects of rupture directivity and shaking hazard, which is dominated by the rare larger earthquakes.

\subsection{Discussion}

[27] We have presented a whole series of measurements for one set of parameters in the model, but a crucial question is how robust these results are to changes in the parameters. 
Two sets of parameters should be distinguished: those related to numerical considerations, such as domain size, and grid resolution and those related to physical considerations, such as frictional parameters. Appendix A discusses the parameters and their values further. We have checked that all of our results are insensitive to numerical parameters. Thus changing domain size does not affect the results. Changing grid resolution when frictional cutoffs stabilize the high frequencies (the friction parameter $\epsilon$ is large enough, see Appendix A) does not affect the results; even then, if this parameter is made too small to provide a cutoff, so that grid resolution instead provides the cutoff, we still end up with qualitatively similar results, with just somewhat higher values for peak acceleration at higher grid resolution, as one would expect.

[28] Changing physical parameters does make quantitative differences in what we see, while still retaining the qualitative picture we have presented thus far. Figure 8 shows some examples of different parameters effects on shaking and epicentral density. We do of course see changes in the amplitudes by changing physical parameters, with for example higher peak accelerations and velocities with increased weakening rate (larger $\alpha$, see Appendix A). Figure 8a illustrates this case of increased weakening rate. Note the similarity with Figure $6 \mathrm{a}$ and the increased amplitude of the color scale. These changes arise from changes in the dynamic ruptures, with faster weakening leading to faster rupture propagation velocities and more rapid slip rates.

[29] In fact, the overall hazard pattern is remarkably robust to friction parameter values. Changing the weakening rate $\alpha$, the timescale for the time weakening mechanism $t_{0}$, and the initial stress drop $\sigma_{0}$ (see Appendix A) all have little effect on the pattern seen in Figures $6 a$ and $8 a$, with the main effect being an overall change in the amplitude. Even changing the frictional instability has remarkably little effect: Figure $8 \mathrm{~b}$ illustrates this as we change to velocity weakening from the previously used slip weakening. Changing $\epsilon$ has a somewhat stronger effect, tending to concentrate the hazard closer to the fault, but the overall pattern in terms of hazard asymmetry along the various segments is little changed.

[30] A bigger effect occurs when we change the physics more substantially. One consequence of the geological physics we have proposed, that faults weaken with slip, is that this effect unfortunately also feeds back into the rupture dynamics, acting as an additional slip weakening term, leading to prolonged risetimes and additional slip in large events. This also makes the ruptures somewhat sluggish, with typical propagation velocities of order 0.7 times the one wave speed in our scalar model. We have checked that this sluggishness in the ruptures does not affect the basic results we have presented by examining another model whereby we begin from the same rupture geometry, and then switch off any additional geological weakening during the long sequence of dynamic events which follow (we accomplish this by setting $\beta=0$ for additional slip after some initial time $t>t_{a}$; see Appendix A). This has the disadvantage that in the very long run, the ruptures will tend to delocalize as stresses accumulate from continued deformation without any compensating geological weakening. However, since we run in the regime where faults evolve only very slightly over many earthquake cycles (as in real earthquakes where it takes hundreds of large events to change geometries appreciable since earthquake strains are of order $10^{-4}$ and fault strains are of order $10^{-2}$ [Scholz, 2002]), this long-term delocalization does not affect appreciably the tens of large event repeats we examine. This regime corresponds to event stress drops being a small fraction of fault strength drops, which again is the relevant regime for earthquakes, where earthquake stress drops are of order a fraction of the overburden stress (few megapascal stress drops) and fault strength drops are of order a fraction of the modulus (tens of thousands of megapascals). Despite the long-term delocalization disadvantage, this has the advantage of producing more realistic ruptures, with typical propagation velocities now more like 0.9 times the wave speed and large event risetimes more like the brittle crust depth, and no extended afterslip on the large events. Figure 8c shows ruptures with this modified fault physics. Now, more substantial changes are seen, with changing patters of ruptures on the fault system. That is, keeping the same fault geometry, we find different sets of ruptures on the faults, so that, for example, a fault which had ruptures predominantly propagating upward, may now have ruptures predominantly propagating downward. Figure $8 \mathrm{c}$ illustrates this case where we see now turning off the additional geological weakening that some of the segments change their shaking asymmetry. Thus note, for example, how on the lower left segment we see a change from the higher shaking at the top to the higher shaking at the bottom. At the same time, the epicenter and directivity origin of the shaking asymmetry pointed out in Figure $7 \mathrm{~b}$ is preserved: When we look at the epicentral density shown in Figure 8 (bottom) for these different friction parameters, we see a corresponding change in where the events are initiating, with the majority of epicenters switching from the top to the bottom of the segment as the stronger shaking switches from the bottom to the top of the segment. This suggests that fault geometry alone is not a sufficient determinant of rupture patters, and dynamics and frictional properties will also need to be understood to get ruptures right. On the other hand, it does further encourage the use of seismicity, which naturally organizes in response to both geometry and frictional properties, to anticipate rupture directivity.

\subsection{Implications}

[31] With an instrumental record which typically spans only a fraction of an earthquake cycle, and with new large events continuing to bring new surprises, we do not have the luxury of waiting to solve the earthquake hazard problem by empirical observations alone. We have presented a physically based model which is both simple enough to be clearly posed, and also is rich enough to have many of the complex behaviors we seek to understand about earthquakes. Measuring directly the shaking hazard and systematics of long sequences of dynamic ruptures, the results we have presented raise a number of questions with important hazard implications.

[32] The differing spatial patterns between the peak acceleration exceedance for a given probability, and the probability of exceedance of a given peak acceleration, and the kinetic energy support the effort to develop fuller "vector" measures of earthquake shaking and damage 

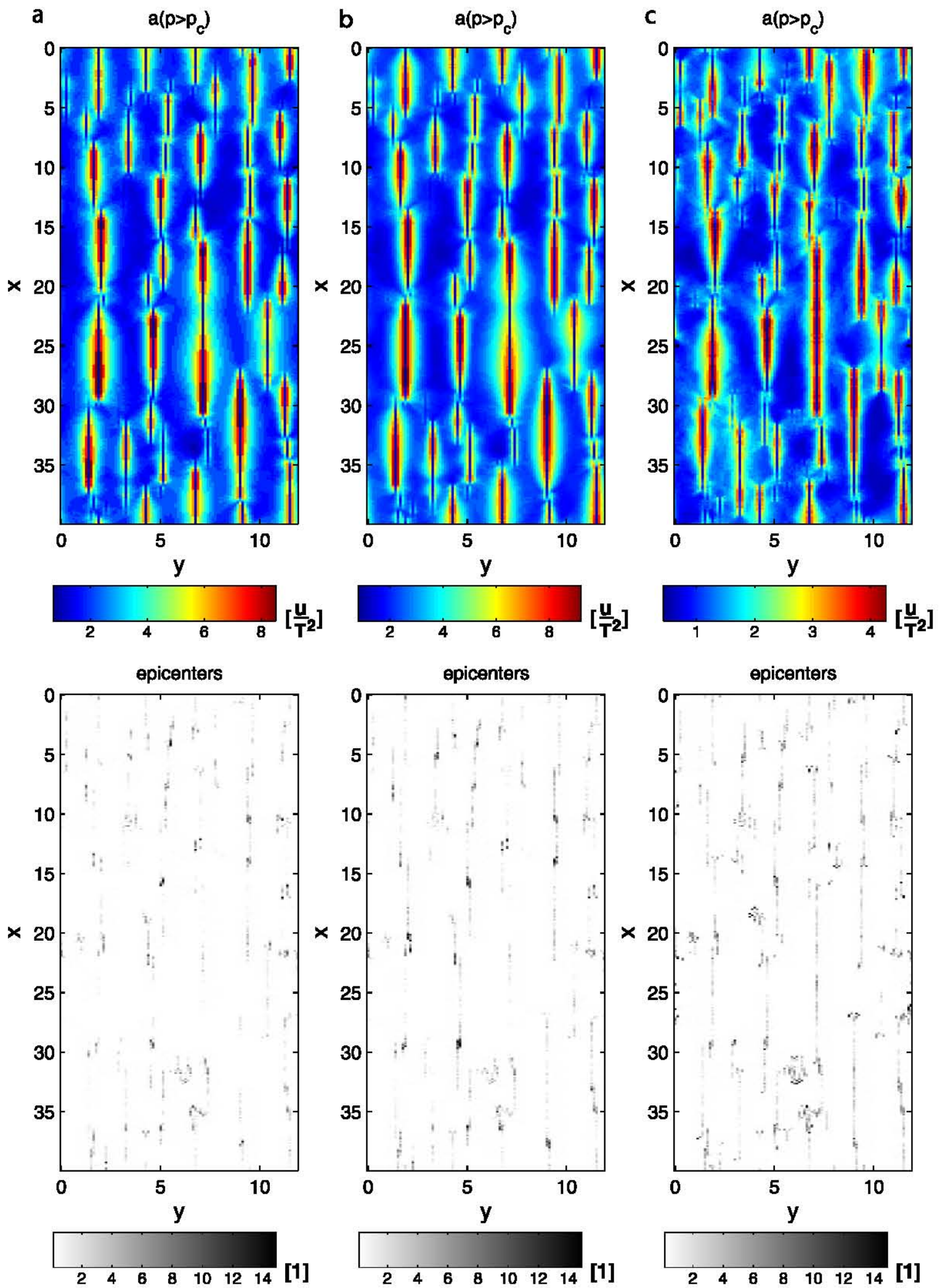

Figure 8. Three different sets of parameters showing change with respect to parameter values. (top) Peak acceleration and (bottom) epicenter densities. Parameter values show: (a) stronger rate of slip weakening, (b) velocity weakening, and (c) geological slip weakening turned off for dynamic event sequence. Note that when shaking asymmetry changes from one panel to the next, epicentral density asymmetry changes as well (see, for example, lower left segment in Figure $8 \mathrm{c}$ as compared to Figures $8 \mathrm{a}$ and $8 \mathrm{~b})$. 
potential; they are not simply related to each other [Baker and Cornell, 2005].

[33] The persistence of the directivity of ruptures seen in the model, where segments often rupture in a certain direction has significant hazard implications if it indeed holds for real faults. One way of examining this in the field would be to look at variations in the existence and density of precarious rocks along segments [Brune et al., 2004]. An additional way of examining this in the field would be through damage asymmetry; while the relationship to directivity may be difficult to quantitatively develop, qualitatively if damage occurs primarily on the side of decreasing normal stress, one would expect segments with high directivity fractions to have more damage on the extensional side. Indeed, damage asymmetry has been observed along faults, and ascribed to such rupture direction asymmetries [Dor et al., 2006].

[34] Finally, the significant correlations we have seen in the model of epicentral location asymmetry with the asymmetry in the peak acceleration shaking hazard, suggests new ways of refining the spatial dependence of shaking hazard. Exploring the observational evidence for these associations, beginning with the connections of epicentral locations and the directivity of recorded large events, is a key next step.

\section{Appendix A: Model Equations}

[35] The equations of motion we are solving are as follows. In the two-dimensional (2-D) scalar bulk, we have

$$
\frac{\partial^{2} u}{\partial t^{2}}=\nabla^{2} u+(w-u)-\eta \frac{\partial u}{\partial t}+\nabla \cdot \mathbf{M}
$$

where $u$ is displacement, $t$ is time,

$$
\nabla^{2}=\frac{\partial^{2}}{\partial x^{2}}+\frac{\partial^{2}}{\partial y^{2}}
$$

is the two-dimensional Laplace operator representing the horizontal elastic coupling of the displacement field, and the $w-u$ term represents the vertical coupling to the lower ductile layer. This layer is slowly stretched, loading the upper brittle layer and moving as

$$
w=v y t
$$

with $v \ll 1$. (Shear loading would correspond with this motion in the $\hat{x}$ direction, while extensional loading would correspond with this motion in the $\hat{y}$ directions; in either case, in our simplified two dimensional scalar model, the results would be the same.) Loading from below distributes deformation throughout the upper layer, as in the Basin and Range province of the Western United States. This length scale (scaled to unity) for the brittle layer depth then sets the horizontal length scale between major faults.

[36] The dissipation constant $\eta$ damps the waves, and is used to mimic geometrical spreading effects which are otherwise much weaker in our 2-D model as compared to 3-D. The final term is the body forces arising from the fault dislocation openings $\mathbf{M}$

$$
\mathbf{M}=\left.\delta u\right|_{\Gamma}
$$

The boundary condition on the faults $\Gamma$ are that the normal strain equals the traction

$$
\left.\nabla u \cdot \perp \Gamma\right|_{\Gamma}=\phi .
$$

[37] All of the nonlinearity in the problem is contained in the friction $\phi$, which has a stick-slip form, resisting motion up to some threshold value, and acting against motion when sliding occurs. We represent the stick slip by

$$
\phi=\Phi\left(\frac{\partial S}{\partial t^{\prime}}, t^{\prime} \leq t\right) H\left(\frac{\partial S}{\partial t}\right)
$$

where $\Phi$ is a scalar frictional strength, $S=|\mathbf{M}|$ is the slip and $\partial S / \partial t$ is the slip rate on the fault, and $H$ is the antisymmetric step function

$$
H= \begin{cases}\frac{\partial S}{\partial t} & \frac{\partial S}{\partial t} \neq 0 \\ |H|<1 & \frac{\partial S}{\partial t}=0 . .\end{cases}
$$

which represents the stick-slip nature of the friction, being multivalued at zero slip rate, and opposing motion in the $\partial S / \partial t$ unit direction when slipping.

[38] What remains a big open question for earthquakes, is what is the frictional strength $\phi$. While there are reasons for thinking we may have a pretty good description of friction at slow slip rates [Dieterich, 1994; Heslot et al., 1994], at high slip rates things are extremely uncertain; many potential physical effects may be occurring, with substantially different implications for friction [Sibson, 1973; Melosh, 1996; Rice, 1999; Tullis and Goldsby, 2003]. With friction at high slip rates being an open question, we use a friction which has a minimum of parameters, is computationally efficient, and spans a range of frictional instabilities, including slip, time, and velocity weakening [Shaw, 1995; Shaw and Rice, 2000]. Specifically, we use a $\Phi$ which combines long-term geological strength $\Phi_{S}$ which weakens with accumulated geological slip [Spyropoulos et al., 2002] and a dynamic strength $\Phi_{Q}$ which weakens during events [Shaw, 1997]

$$
\Phi=\Phi_{S}+\Phi_{Q} .
$$

The way the strength $\Phi$ changes, with $\operatorname{slip} S$ and slip rate $\partial S / \partial t$ and other state-dependent variables, can dramatically alter what kinds of faults grow and what kinds of dynamic events occur on the faults. We focus on strength changes which seem physically plausible, give interesting fault localization and dynamic stick-slip events, are relatively simple mathematically, and are numerically efficient. The reader does not need to understand the details of the friction to understand the results of the paper. The equations are presented here for completeness, and so others could reproduce the results.

[39] The long-term strength is given by

$$
\Phi_{S}=\Phi_{0}+\xi-\frac{\beta S}{1+a S} .
$$


Here $\Phi_{0}$ is a constant overall strength which is irrelevant to the problem, and $\xi$ is a random variable of amplitude between 0 and $\xi_{0}$, with equal probabilities distributed over that interval, varying in space but fixed in time. This parameter seeds some initial uncorrelated random strength heterogeneity in the model. Geological slip weakening occurs with the last term, which is proportional to slip $S$ with a constant $\beta . \beta$ affects the degree of localization in the problem, and therefore the resulting fault geometry. For large $\Phi_{0}$, we can operate in a regime where the saturating term $a$ is small and irrelevant. The brittle strain excess $\varepsilon \equiv$ $\left(\nu t-\Phi_{0}\right) / \xi_{0}$ gives the relevant strain [Spyropoulos et al., 2002], so the amplitude $\xi_{0}$ of the uncorrelated random strength heterogeneity scales out of the problem.

[40] For the dynamic strength weakening, we consider three terms

$$
\Phi_{Q}=-\frac{\alpha Q}{1+\alpha Q}-\Sigma_{t}-\epsilon \nabla_{\|}^{2} \frac{\partial S}{\partial t} .
$$

The first term, which is a function of heat $Q$, models frictional weakening from frictional heating; pore fluid effects [Sibson, 1973; Lachenbruch, 1980; Shaw, 1995] and flash heating of asperities [Rice, 1999] are two potentially relevant physical mechanisms which this simplified quantification could represent. The weakening rate constant $\alpha$ is a critical parameter in many aspects of the dynamics, although the results we present here are mainly insensitive to it. Heat accumulates with slip rate and dissipates over some timescale $1 / \gamma$ :

$$
\frac{\partial Q}{\partial t}=-\gamma Q+\left|\frac{\partial S}{\partial t}\right|
$$

Slip weakening results from $\gamma \ll 1$, while velocity weakening results from $\gamma \gg 1$ [Shaw, 1995; Shaw and Rice, 2000].

[41] The second term in equation (A9)

$$
\Sigma_{t}= \begin{cases}\sigma_{0} \frac{t-t_{s}}{t_{0}} & t-t_{s}<t_{0} \\ \sigma_{0} & t-t_{s} \geq t_{0}\end{cases}
$$

is a nucleation term, for which we make a big simplification by using a time weakening term. It weakens with time $t$ over a timescale $t_{0}$ since beginning slipping at $t_{s}$, and restrengthens when resticking occurs. This allows for a huge numerical speedup compared with more expensive rate and state formulations, both by compressing the nucleation phase into the finite timescale $t_{0}$ and allowing the limit of loading rate $\nu=0$ to be taken. It also, as well, allows the study of time weakening friction. It is not, however, without cost, and short time correlations between events such as aftershocks are not accounted for by this friction. Nevertheless, it does allow for our numerical time costs to be dominated by the regime of most interest to long-term shaking hazard, the dynamic rupture timescale.

[42] The last term $\epsilon \nabla_{\|}^{2}(\partial S / \partial t)$, with $\epsilon$ a small constant and $\nabla_{\|}^{2}$ the fault parallel second derivative, provides stability at the shortest wavelengths [Langer and Nakanishi, 1993; Shaw and Rice, 2000].

[43] For numerical simplicity, we restrict the fault segments $\Gamma$ to be perpendicular to the loading direction $y$, set by equation (A2). We also discretize the equations onto a rectangular grid, and use a second-order finite difference approximation of the continuum equations. The numerical scheme proceeds by first evolving the fault system quasistatically, taking advantage of the dependence of the fault system evolution on the total slip, rather than slip increments, on the faults. Previous work has shown the fault system which evolves is insensitive to the details of the slip increments, since the interactions occur through the total slip on the faults [Spyropoulos et al., 2002]. This allows us to evolve rapidly through geological time. Once a desired total strain is reached, the system is switched to elastodynamic mode. Now all slip increments occur elastodynamically. We choose the initial strain to be large relative to the strain which accumulates during the long elastodynamic catalogue, so the fault system remains little changed by the dynamic events, the geometry being dominated by the long previous geological history. The system is loaded until one point is just at the point of failure. The event evolves then under fully inertial dynamics. Once the event has stopped slipping, the waves are quenched in the system; then the system is reloaded until the next point is just at failure. This reloading is accomplished easily by calculating how far the static solution is from failure at every point, and then loading so the least stable point is just at failure. For simple geometries and frictions such as we have, this loading can be done analytically, while for more complicated geometries and frictions, numerical Green's functions can be used.

[44] Parameters used in the simulations shown, unless otherwise indicated, are fault parameters $\beta=0.4, \varepsilon=1.0$; domain parameters $\delta_{x}=0.125, \delta_{y}=0.125, L_{x}=40, L_{y}=12$; bulk parameter $\eta=0.3$; friction parameters $\alpha=3, \gamma=0.1$, $\sigma_{0}=0.3, t_{0}=0.2, \epsilon=0.003$. Typical catalogue lengths are $v t \sim 100$ so that tens of repeat times of large events are simulated, corresponding to timescale of order thousands of years. Typical numbers of events in the catalogues are thousands to tens of thousand, with a wide range of event sizes.

[45] The simulation is run using dimensionless units, so as to minimize the number of parameters. For use in comparing with the real earthquake system, however, we can also covert back to dimensional variables. The conversion back to dimensional units is as follows:

$$
\begin{aligned}
\text { wave speed } & c=1=L / T=3 \mathrm{~km} / \mathrm{s} \\
\text { crustal length scale } & L=1=15 \mathrm{~km} \rightarrow \\
\text { crustal timescale } & T=1=5 \mathrm{~s} \\
\text { stress drop } & \delta \phi=1=u G / L=3 \mathrm{MPa} \rightarrow \\
\text { large event slip } & u=1=\delta \phi L / G=10^{-4} L= \\
& 1.5 \mathrm{~m} \\
\text { modulus } & G=3 \times 10^{4} \mathrm{Mpa} \\
\text { slip rate } & \pi u / T=\pi=1.0 \mathrm{~m} / \mathrm{s} \\
\text { acceleration } & \pi^{2} u / T^{2}=\pi^{2}=0.06 \mathrm{~m} / \mathrm{s}^{2}=0.05 \mathrm{~g} \\
\text { slip-weakening length } & u / \alpha=0.5 \mathrm{~m} \\
\text { frictional healing time } & 1 / \gamma=10 T=50 \mathrm{~s} \\
\text { domain size along faults } & L_{x}=40 L=600 \mathrm{~km} \\
\text { domain size } \perp \text { faults } & L_{y}=12 L=180 \mathrm{~km} \\
\text { grid resolution } & \delta_{x}=0.125 L \approx 2 \mathrm{~km} \\
\text { Nyquist frequency } & c / 2 \delta_{x} \approx 1 \mathrm{~Hz}
\end{aligned}
$$

[46] Acknowledgments. This work was supported by NSF grants EAR-02-29834 and EAR-03-37226 and by a grant from the Southern 
California Earthquake Center. Martin Mai and an anonymous reviewer provided helpful comments.

\section{References}

Aochi, H., E. Fukuyama, and M. Matsu'ura (2000), Selectivity of spontaneous rupture propagation on a branched fault, Geophys. Res. Lett., 27, 3635.

Baker, J. W., and C. A. Cornell (2005), A vector-valued ground motion intensity measure consisting of spectral acceleration and epsilon, Earthquake Eng. Struct. Dyn., 34, 1193.

Bouchon, M., and D. Streiff (1997), Propagation of a shear crack on a nonplanar fault: A method of calculation, Bull. Seismol. Soc. Am., 87, 61.

Brune, J. N. (2001), Shattered rock and precarious rock evidence for strong asymmetry in ground motions during thrust faulting, Bull. Seismol. Soc. Am., 91, 443.

Brune, J. N., A. Anooshehpoor, B. P. Shi, and Y. H. Zeng (2004), Precarious rock and overturned transformer evidence for ground shaking in the $M_{s} 7.7$ Kern County earthquake: An analog for disastrous shaking from a major thrust fault in the Los Angeles basin, Bull. Seismol. Soc. Am., 94, 1993.

Carlson, J. M., and J. S. Langer (1989), Mechanical model of an earthquake fault, Phys. Rev. A, 84, 6470 .

Cochard, A., and R. Madariaga (1996), Complexity of seismicity due to highly rate-dependent friction, J. Geophys. Res., 101, 25,331.

Dawers, N. H., and M. H. Anders (1995), Displacement-length scaling and fault linkage, J. Struct. Geol., 17, 604.

Dieterich, J. H. (1994), A constitutive law for the rate of earthquake production and its application to earthquake clustering, J. Geophys. Res., 99, 2601.

Dor, O., T. K. Rockwell, and Y. Ben-Zion (2006), Geologic observations of damage asymmetry in the structure of the San Jacinto, San Andreas and Punchbowl faults in southern California: A possible indicator for preferred rupture propagation direction, Pure Appl. Geophys., 163, 301349, doi:10.1007/s00024-005-0023-9.

Duan, B., and D. D. Oglesby (2005), Multicycle dynamics of nonplanar strike-slip faults, J. Geophys. Res., 110, B03304, doi:10.1029/ 2004JB003298.

Fliss, S., H. S. Bhat, R. Dmowska, and J. R. Rice (2005), Fault branching and rupture directivity, J. Geophys. Res., 110, B06312, doi:10.1029/ 2004JB003368.

Frankel, A., et al. (2000), USGS national seismic hazard maps, Earthquake Spectra, 16, 1.

Graizer, V., and A. Shakal (2004), Attenuation and directivity of peak ground acceleration (pga) in near fault strong motion from M6.0 Parkfield, California earthquake, Eos Trans. $A G U, 85(47)$, Fall Meet. Suppl., Abstract S51C-01700.

Harris, R. A., R. J. Archuletta, and S. M. Day (1991), Fault steps and the dynamic rupture process: 2-D numerical simulations of a spontaneously propagating shear fracture, Geophys. Res. Lett., 18, 893.

Heslot, F., T. Baumberger, B. Perrin, B. Caroli, and C. Caroli (1994), Creep, stick-slip, and dry-friction dynamics-Experiments and a heuristic model, Phys. Rev. E, 49, 4973.

Kame, N., and T. Yamashita (1997), Dynamic nucleation process of shallow earthquake faulting in a fault zone, Geophys. J. Int., 128, 204.

King, G. C. P. (1986), Speculations on the geometry of the initiation and termination processes of earthquake rupture and its relation to morphology and geological structure, Pure Appl. Geophys., I24, 567.

King, G. C. P., and J. Nabelek (1985), The role of bends in faults in the initiation and termination of earthquake rupture: Implications for earthquake prediction, Science, $228,984$.

Kossobokov, V. G., and J. M. Carison (1995), Active zone size versus activity: A study of different seismicity patterns in the context of the prediction algorithm M8, J. Geophys. Res., 100, 6431.

Lachenbruch, A. (1980), Frictional heating, fluid pressure, and the resistance to fault motion, J. Geophys. Res., 85, 6097.

Langer, J. S., and H. Nakanishi (1993), Models of rupture propagation II: Two dimensional model with dissipation on the fracture surface, Phys. Rev. $E, 48,439$.
Lyakhovsky, V., Y. Ben-Zion, and A. Agnon (2001), Earthquake cycle, fault zones, and seismicity patterns in a rheologically layered lithosphere, J. Geophys. Res., 106, 4103.

Manighetti, I. G. C. P. King, Y. Gaudemer, C. H. Scholz, and C. Doubre (2001), Slip accumulation and lateral propagation of active normal faults in Afar, J. Geophys. Res., 106, 13,667.

Manighetti, I., M. Campillo, C. Sammis, P. M. Mai, and G. C. P. King (2005), Evidence for self-similar, triangular slip distributions on earthquakes: Implications for earthquake and fault mechanics, $J$. Geophys. Res., 110, B05302, doi:10.1029/2004JB003174.

McGuire, J. J., L. Zhao, and T. H. Jordan (2002), Predominance of unilateral rupture for a global catalog of large earthquakes, Bull. Seismol. Soc. Am., 92, 3309.

Melosh, H. J. (1996), Dynamical weakening of faults by acoustic fluidization, Nature, 379, 601 .

Mora, P., and D. Place (1999), The weakmess of earthquake faults, Geophys. Res. Lett., 26, 123.

Myers, C. H., B. E. Shaw, and J. S. Langer (1996), Slip complexity in a crustal plane model of an earthquake fault, Phys. Rev. Lett., 77, 972.

Oglesby, D. D., R. J. Archuleta, and S. B. Nielsen (1998), Earthquakes on dipping faults: The effects of broken symmetry, Science, 280, 1055.

Pepke, S. L., J. M. Carlson, and B. E. Shaw (1994), Prediction of large events on a dynamical model of a fault, J. Geophys. Res., 99, 6769.

Rice, J. R. (1999), Flash heating at asperity contacts and rate-dependent friction, Eos Tran. AGU, 80(46), Fall Meet. Suppl., F6811.

Scholz, C. H. (1997), Size distributions for large and small earthquakes, Bull. Seismol. Soc. Am., 87, 1074.

Scholz, C. H. (2002), The Mechanics of Earthquakes and Faulting, 2nd ed., Cambridge Univ. Press, New York.

Shaw, B. E. (1995), Frictional weakening and slip complexity on earthquake faults, $J$. Geophys. Res., 100, 18,239.

Shaw, B. E. (1997), Model quakes in the two-dimensional wave equation, J. Geophys. Res., 102, 27,367.

Shaw, B. E. (2004a), Self-organizing fault systems and self-organizing elastodynamic events on them: Geometry and the distribution of sizes of events, Geophys. Res. Lett., 31, L17603, doi:10.1029/2004GL019726.

Shaw, B. E. (2004b), Variation of large elastodynamic earthquakes on complex fault systems, Geophys. Res. Lett., 31, L18609, doi:10.1029/ 2004 GL019943.

Shaw, B. E., and J. R. Rice (2000), Existence of continuum complexity in the elastodynamics of repeated fault ruptures, J. Geophys. Res., 105, 23,791.

Sibson, R. H. (1973), Interactions between temperature and pore fluid pressure during earthquake faulting and a mechanism for partial or total stress relief, Nature Phys. Sci., 243, 66 .

Somerville, P. G., N. F. Smith, R. W. Graves, and N. A. Abrahamson (1997), Modification of empirical strong ground motion attenuation relations to include the amplitude and duration of effects of rupture directivity, Seismol. Res. Lett., 68, 199.

Spyropoulos, C., W. J. Griffith, C. H. Scholz, and B. E. Shaw (1999), Experimental evidence for different strain regimes of crack populations in a clay model, Geophys. Res. Lett., 26, 1081.

Spyropoulos, C., C. H. Scholz, and B. E. Shaw (2002), Transition regimes for growing crack populations, Phys. Rev. $E, 65,056105$.

Stirling, M. W., S. G. Wesnousky, and K. Shimazaki (1996), Fault trace complexity, curmulative slip, and the shape of the magnitude-frequency distribution for strike-slip faults: A global survey, Geophys. J. Int., 124, 833.

Tullis, T. E., and D. L. Goldsby (2003), Flash melting of crustal rocks at almost seismic slip rates, Eos Tran. $A G U, 84(46)$, Fall Meet. Suppl., Abstract S51B-05.

Ward, S. N. (1997), Dogtails versus rainbows: Synthetic earthquake rupture models as an aid in interpreting geological data, Bull. Seismol. Soc. Am., $87,1422$.

B. E. Shaw, Lamont-Doherty Earth Observatory, Route 9W, Palisades, NY 10964, USA. (shaw@Ideo.columbia.edu) 